University of Nebraska - Lincoln

DigitalCommons@University of Nebraska - Lincoln

February 1994

\title{
The nuclear hexadecapole interaction of iodine-127 in cadmium iodide measured using zero-field two dimensional nuclear magnetic resonance
}

\author{
Ming-Yuan Liao \\ University of Nebraska - Lincoln
}

Gerard S. Harbison

University of Nebraska - Lincoln, gharbison1@unl.edu

Follow this and additional works at: https://digitalcommons.unl.edu/chemistryharbison

Part of the Chemistry Commons

Liao, Ming-Yuan and Harbison, Gerard S., "The nuclear hexadecapole interaction of iodine-127 in cadmium iodide measured using zero-field two dimensional nuclear magnetic resonance" (1994). Gerard Harbison Publications. 3.

https://digitalcommons.unl.edu/chemistryharbison/3

This Article is brought to you for free and open access by the Published Research - Department of Chemistry at DigitalCommons@University of Nebraska - Lincoln. It has been accepted for inclusion in Gerard Harbison Publications by an authorized administrator of DigitalCommons@University of Nebraska - Lincoln. 


\title{
The nuclear hexadecapole interaction of iodine-127 in cadmium iodide measured using zero-field two dimensional nuclear magnetic resonance
}

\author{
Ming-Yuan Liao and Gerard S. Harbison \\ Department of Chemistry, University of Nebraska at Lincoln, Lincoln, Nebraska 68588-0304
}

(Received 25 June 1993; accepted.18 October 1993)

\begin{abstract}
Two dimensional nuclear quadrupole correlation spectroscopy has been used to measure simultaneously the two nuclear spin transition frequencies for the iodine resonance of cadmium iodide at zero magnetic field. Because of the layer structure and resultant polymorphism in this substance, conventional zero-field NMR spectra are inhomogeneously broadened by several hundred $\mathrm{kHz}$. However, the 2D spectra obtained by our method are subject only to homogeneous linewidth, which for this compound is of the order of $5 \mathrm{kHz}$. This greatly increased precision allows more accurate evaluation of the nuclear hexadecapole coupling in cadmium iodide; which we measure as $0.216 \pm 0.514 \mathrm{kHz}$ (i.e., indistinguishable from zero). This gives a maximum value of the nuclear hexadecapole moment several times lower than that recently reported for the same nucleus in potassium iodide.
\end{abstract}

\section{INTRODUCTION}

Of all the fundamental electrostatic interactions of ordinary matter, the nuclear hexadecapole coupling is perhaps the most ephemeral. Since it was reported in $1955,{ }^{1}$ it has gone through cycles of measurement ${ }^{2-4}$ and refutation; $;$,6 almost 40 years after its initial appearance in the literature, its very existence as a nonzero quantity is still somewhat in doubt. Perhaps the most rigorous recent work on the subject, by Doering and Waugh in $1986,{ }^{5}$ showed that most of the measurements up to that point in time were within experimental uncertainty of zero; they themselves were unable to determine a nonzero hexadecapole interaction in a tantalate salt due to residual line broadening.

Since hexadecapolar interactions themselves, if they are measurable at all, are certainly very small, they have until now been measured as perturbations on some much large interaction which provides the Boltzmann distribution and increased resonance frequency neccssary for efficient detection. The two main approaches have been to measure the spectrum at zero field, in the presence of a quadrupolar interaction (conventionally but not completely accurately called NQR spectroscopy); this requires measurement of three independent transition frequencies, or of two frequencies in systems where the lattice site symmetry is no lower than axial -or at high field in a site of cubic point symmetry, in which the thermal nuclear polarization results from the Zeeman interaction, and the quadrupolar interaction is theoretically zero.

The two methods have had significant drawbacks: The hexadecapolar interaction at zero field is manifest as a perturbation of the ratios of two (in the case of axial symmetry) or three (in the general case) quadrupolar-induced spin transition frequencies. Both transitions are often subject to significant inhomogeneous line width, often tens or hundreds of $\mathrm{kHz}$. In addition, if the two or three frequencies are measured sequentially, changes in experimental conditions between the time of the measurements may induce a change in the ratio of frequencies which may be spuriously interpreted as due to a hexadecapole. The highfield NMR method is limited by the fact that the electric field gradient at the nuclear site is never identically zero in any real crystal, regardless of symmetry; even in the most perfect crystal, strain and residual imperfections cause the NMR linewidth to be inhomogeneously broadened, just as it is in the quadrupolar case. In addition, if the quadrupolar interactions are large enough, they may enter the second-order regime; a second rank perturbation on a first rank tensor has fourth rank components in the second order of approximation; these fourth rank components have the same symmetry properties as the first order part of the hexadecapole perturbation and so may mimic them closely.

Since one of the goals of the high field method-to choose the point symmetry of the lattice site to eliminate the quadrupolar interaction - can only be achieved, even in ideal circumstances, for a limited number of materials, and is almost never attainable in practice, the zero-field method, where only two terms need be considered, appears to hold the most promise. The two major drawbacks of the zero-field method, inhomogeneous linewidth and nonsimultaneity of measurements-may both be circumvented by using two-dimensional correlation spectroscopy, which has recently been applied by us to quadrupolar nuclei at zero field. ${ }^{7}$ We report here the utilization of these methods to measure with unprecedented precision the hexadecapolar interaction of ${ }^{127} \mathrm{I}$ in cadmium iodide; setting an upper limit on the Sternheimer-weighted nuclear hexadecapole moment of this nucleus several times lower than previous measurements. ${ }^{6}$

\section{THEORY}

\section{Hexadecapolar perturbation of the zero-field NMR spectrum}

For a nucleus at the zero field, the quadrupole Hamiltonian is given by

$$
H_{q}=\frac{e^{2} Q q}{4 I(2 I-1)}\left[\left(3 \mathbf{I}_{z}^{2}-\mathbf{I} \cdot \mathbf{I}\right)+\frac{\eta}{2}\left(\mathbf{I}_{+}^{2}+\mathbf{I}_{-}^{2}\right)\right] .
$$


Conventionally $I$ represents the spin of a nucleus, $e Q$ is the quadrupole moment for the nucleus, $e q$ is the second derivative of the electric potential at the nuclear site. $\eta$ is the asymmetry parameter, and $e^{2} Q q$ is the quadrupole coupling constant (QCC). Similarly, the Hamiltonian for the diagonal $\left(T_{40}\right)$ part of the hexadecapole interaction can be written as

$$
\begin{aligned}
H_{h}= & \frac{e^{2} H h}{128 I(I-1)(2 I-1)(2 I-3)}\left[35 I_{z}^{4}-30 I_{z}^{2}(I \cdot I)\right. \\
& \left.+3(\mathbf{I} \cdot \mathbf{I})^{2}+25 I_{z}^{2}-6(I \cdot \mathbf{I})\right]
\end{aligned}
$$

where $e H$ is the hexadecapole moment of the nucleus and $e h$ is the fourth derivative of the electric potential at the nucleus site. We define $e^{2} H h$ as the hexadecapole coupling constant (HCC).

For a spin $5 / 2$ system with axial symmetry, the measured NQR frequencies perturbed by HDI can be written as

$$
\begin{aligned}
& v_{1}\left(\frac{5}{2}-\frac{3}{2}\right)=\frac{12}{40} \mathrm{QCC}+\frac{12}{192} \mathrm{HCC}, \\
& v_{2}\left(\frac{3}{2}-\frac{1}{2}\right)=\frac{6}{40} \mathrm{QCC}-\frac{15}{192} \mathrm{HCC} .
\end{aligned}
$$

Therefore, the quadrupole coupling constant QCC and hexadecapole coupling constant HCC can be calculated by

$$
\begin{aligned}
& \mathrm{QCC}=\frac{10}{21}\left(5 v_{1}+4 v_{2}\right), \\
& \mathrm{HCC}=\frac{32}{7}\left(v_{1}-2 v_{2}\right) .
\end{aligned}
$$

From the experimental quadrupole and hexadecapole coupling constants, using the Sternheimer relationship, the electric field gradient and the second derivative of the electric field gradient around the ion site can be calculated using

$$
\begin{aligned}
& e q=\frac{\mathrm{QCC}}{\left(1-\gamma_{\infty}\right) e Q}, \\
& e h=\frac{\mathrm{HCC}}{\left(1-\eta_{\infty}\right) e H},
\end{aligned}
$$

where $\gamma_{\infty}$ and $\eta_{\infty}$ are the Sternheimer factors for the quadrupole and hexadecapole moment.

\section{Calculation of the electric field gradient and its second derivative}

If there exists an electric field $\mathbf{E}$ at the site of a closedshell ion, the induced electric dipole moment is given by $\mathbf{P}=\alpha_{d} \mathbf{E}$, where $\alpha_{d}$ is the dipolar polarizability of the ion. Similarly, the electric field gradient $\nabla E$ will produce an induced electric quadrupole moment which is given by $\mathbf{Q}=\alpha_{q} \mathbf{\nabla E}$, where $\alpha_{q}$ is the quadrupolar polarizability of the ion. Therefore, the total electric field at the origin due to an object at point $\mathbf{r}$ may be expressed in terms of the moments of the distribution

$$
\mathbf{E}(\mathbf{r})=\frac{q}{4 \pi \epsilon_{0}} \frac{\hat{\mathbf{r}}}{r^{2}}+\frac{\mathbf{P}}{4 \pi \epsilon_{0}} \frac{1-3 \hat{\mathbf{r}} \hat{\mathbf{r}}}{r^{3}}+\frac{\mathbf{Q}}{8 \pi \epsilon_{0}} \frac{5 \hat{\mathbf{r}} \hat{\mathbf{r}} \hat{\mathbf{r}}-2 \hat{\mathbf{r}}}{r^{4}}+\cdots,
$$

where $q$ is the monopole moment due to the point charge, $\mathbf{P}$ is the induced dipole moment due to the electric field at $\mathbf{r}$, and $\mathbf{Q}$ is the induced quadrupole moment due to the electric field gradient at that point. By using standard vector calculus methods we can derive the formula

$$
\nabla \frac{\hat{\mathbf{r}}^{n}}{r_{r}^{m}}=\frac{-1}{r^{m+1}}\left[(m+n) \hat{\mathbf{r}}^{n+1}-n \hat{\mathbf{r}}^{n-1}\right] .
$$

In an ionic crystal, the total electric field gradient would be the summation of the electric field gradient due to the monopole, induced dipole and induced quadrupole

$$
\begin{aligned}
\boldsymbol{\nabla E}(\mathbf{r})= & \frac{-q}{4 \pi \epsilon_{0}} \frac{3 \hat{\mathbf{r}} \hat{\mathbf{r}}-1}{r^{3}}+\frac{\mathbf{P}}{4 \pi \epsilon_{0}} \frac{15 \hat{\mathbf{r}} \hat{\mathbf{r}}-9 \hat{\mathbf{r}}}{r^{4}} \\
& +\frac{-\mathbf{Q}}{8 \pi \epsilon_{0}} \frac{35 \hat{\mathbf{r}} \hat{\mathbf{r}} \hat{\mathbf{r}}-25 \hat{\mathbf{r}} \hat{\mathbf{r}}+2}{r^{4}}+\cdots
\end{aligned}
$$

Similarly, the first three terms of the expansion of the second derivative of the electric field gradient are

$$
\begin{aligned}
& \nabla^{3} \mathbf{E}(\mathbf{r})=-\frac{1}{4 \pi \epsilon_{0}} \frac{q}{r^{3}}(105 \hat{\mathbf{r}} \hat{\mathbf{r}} \mathbf{r} \hat{\mathbf{r}}-90 \hat{\mathbf{r}} \hat{\mathbf{r}}+9)+\frac{1}{4 \pi \epsilon_{0}} \frac{\mathbf{P}}{r^{6}} \\
& \times(945 \hat{\mathbf{r}} \hat{\mathbf{r}} \hat{\mathbf{r}} \hat{\mathbf{r}} \hat{\mathbf{r}}-1050 \hat{\mathbf{r}} \hat{\mathbf{r}} \hat{\mathbf{r}}+225 \hat{\mathbf{r}})-\frac{1}{8 \pi \epsilon_{0}} \frac{\mathbf{Q}}{r^{7}}
\end{aligned}
$$

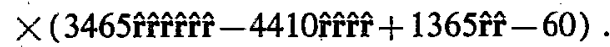

\section{EXPERIMENT}

Cadmium iodide $(99.5 \%)$ was obtained from Johnson Mathey Electronics. Samples for NMR were obtained by cooling a saturated solution of the compound at $40^{\circ} \mathrm{C}$ down to $4{ }^{\circ} \mathrm{C}$ over a $24 \mathrm{~h}$ period in the absence of light; the soft, platelike crystals were separated from the mother liquor by careful filtration and air dried. They were inserted into a glass sample tube without crushing or applying any avoidable stress, and were packed by carefully agitating the tube and allowing them to settle gravitationally. The double-resonance probe, and detailed experimental protocols for the two dimensional experiments, are described previously. ${ }^{7}$ The experiment was carried out using a homebuilt pulsed solid-state NMR spectrometer at zero field. The temperature was kept at $23 \pm 0.5^{\circ} \mathrm{C}$ by air cooling. For one dimensional spectra, a simple $(\pi / 2-\tau-\pi-\tau)$ Hahnecho sequence was used. A series of two dimensional experiments were carried out over the range where $\mathrm{CdI}_{2}$ shows significant signal intensity: i.e., for $v_{1}(5 / 2-3 / 2)$ $=29.600-29.420 \mathrm{MHz}$ and $v_{2}(3 / 2-1 / 2)=14.800-14.710$ MHz. The $90^{\circ}$ pulse lengths, based on the maximum nutation frcquency observed for a powder sample, were $3.5 \mu \mathrm{s}$ for the $(5 / 2-3 / 2)$ transition and $3.0 \mu$ s for the $(3 / 2-1 / 2)$ transition. The basic pulse sequence for two dimensional pure quadrupole correlation spectroscopy (NQ-COSY) is shown in Fig. 1. Two dimensional correlation spectra were acquired by signal averaging 4096 acquisitions using a $\tau$ value of $30 \mu \mathrm{s}$, and a recycle delay of $100 \mathrm{~ms} .128$ free induction decays are collected for both real and imaginary quadrature components in the first dimension, with a $t_{1}$ 


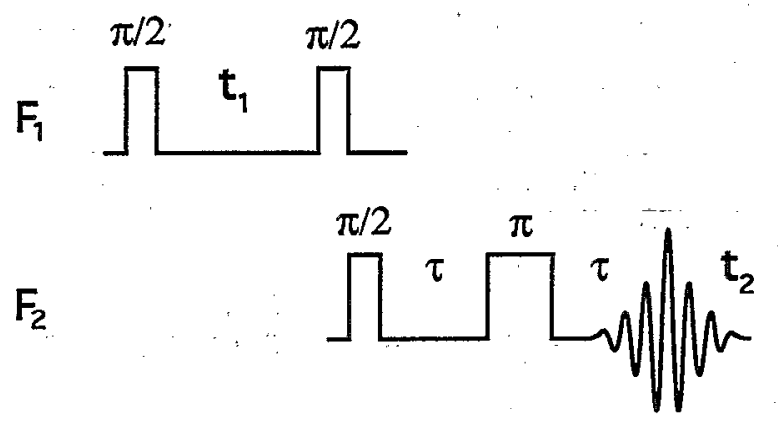

FIG. 1. The NQ-COSY pulse sequence used in the present work.

increment of $2 \mu \mathrm{s}$. The free induction decays provided the second dimension, which contain 512 points at $2 \mu$ s per point.

Figure 2 shows typical one dimensional spectra of ${ }^{127} \mathbf{I}$ in cadmium iodide. The lines are inhomogeneously broadened, presumably as a result of a combination of stacking disorder and polymorphism. In the displayed spectra the width of the $(5 / 2-3 / 2)$ transition is approximately $85 \mathrm{kHz}$ and the $(3 / 2-1 / 2)$ transition $75 \mathrm{kHz}$. These are, however, almost certainly just the excitation bandwidths; they are in good agreement with the expected effective spectral widths for an echo sequence with the r.f. field strengths employed. Furthermore, offsetting the spectrometer frequencies shows the real linewidths to be considerably greater than those observed here. In point of fact, we measure some spectral intensity over the entire region of frequency space spanned by the two reported iodine resonance frequencies of 14.3 and $14.7 \mathrm{MHz}^{8}$

Figure 3 shows a typical two dimensional quadrupole correlation spectrum ${ }^{127} \mathrm{I}$ in cadmium iodide, displayed as
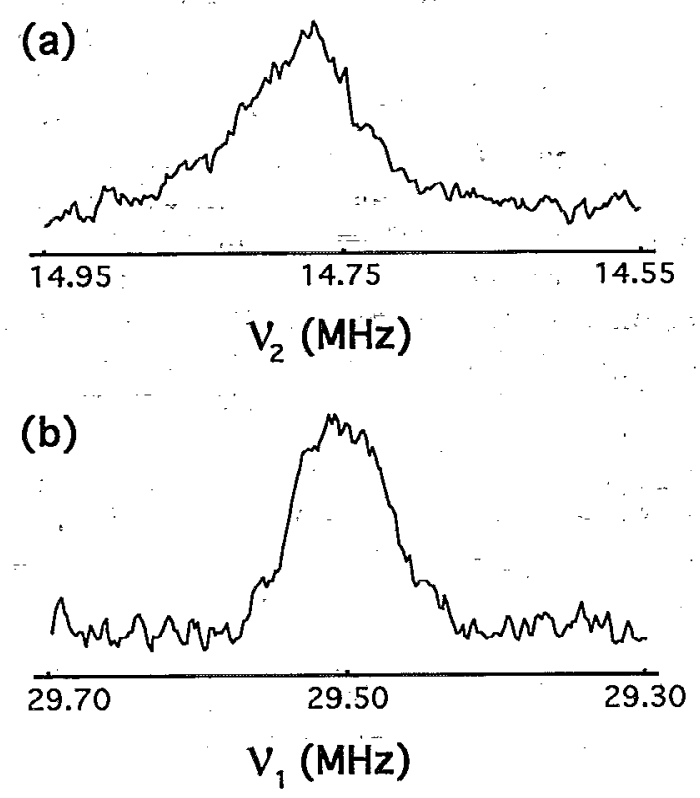

FIG. 2. The zero-field NMR spectra of ${ }^{127} \mathrm{I}$ in cadmium iodide. (a) The $(3 / 2-1 / 2)$ transition. (b) The $(5 / 2-3 / 2)$ transition.
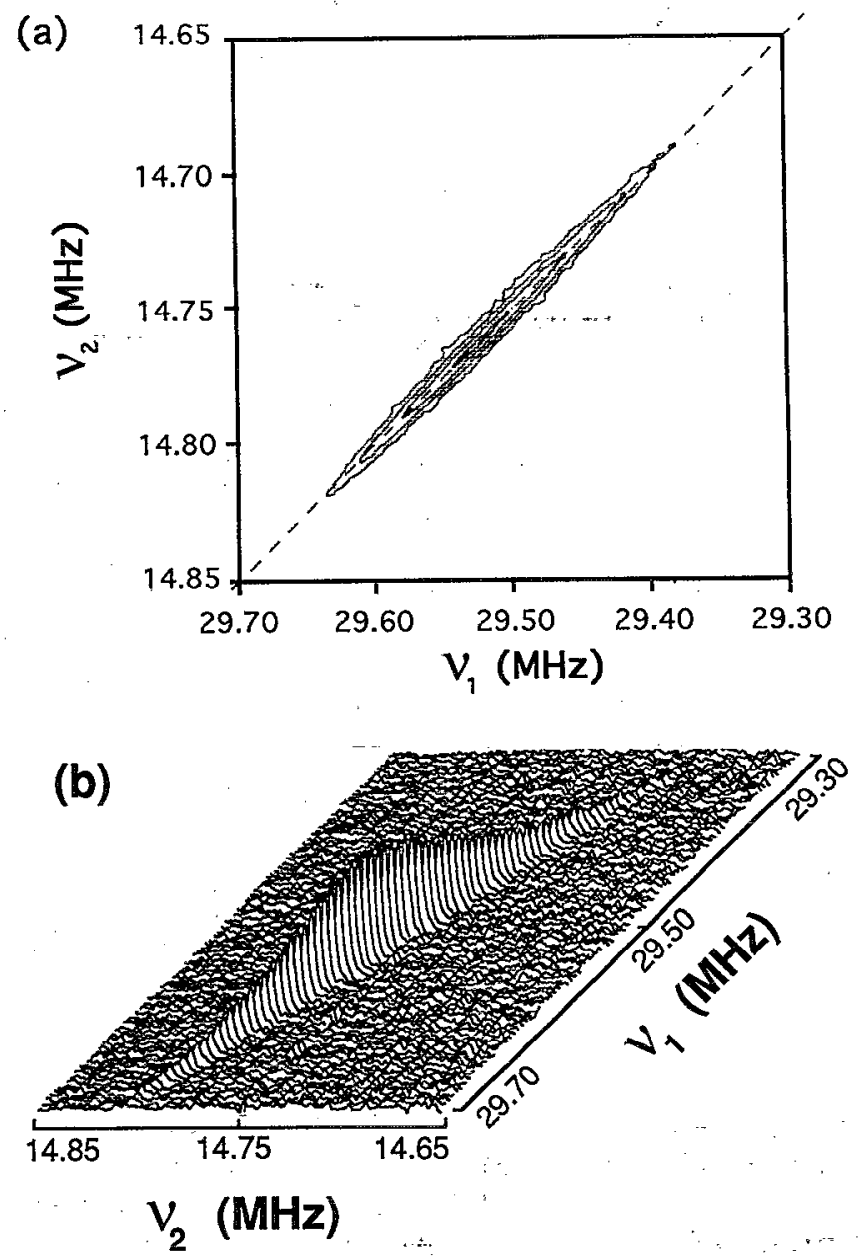

FIG. 3. (a) Contour plot of the two dimensional zero-field NMR correlation spectrum of ${ }^{127} \mathrm{I}$ in cadmium iodide. (b) Stacked plot of the two dimensional zero-field NMR correlation spectrum of ${ }^{127} I$ in cadmium iodide. These spectra were obtained by the NQ-COSY pulse sequence shown in Fig. 1.

both contour and stacked plots. The observation frequencies in the $v_{1}(5 / 2-3 / 2)$ and $v_{2}(3 / 2-1 / 2)$ dimensions were 29.500 and $14.750 \mathrm{MHz}$, respectively. The inhomogeneous linewidth now extends along a diagonal of slope 2 , and is as expected, about $80 \mathrm{kHz}$ in the $v_{1}$ dimension. It is therefore entirely absent from slices parallel to either frequency axis (Fig. 4 shows two typical intersecting slices); these are subject only to the homogeneous linewidths, which are $11.2 \mathrm{kHz}$ for the $(5 / 2-3 / 2)$ transition and $5.5 \mathrm{kHz}$ for the $(3 / 2-1 / 2)$ transition. The fact that the ratios of linewidths are almost exactly identical to the ratios of frequencies is amusing but of uncertain import. The precise ratios of frequencies over a $100 \mathrm{kHz}$ region of the diagonal "spine" were determined by fitting 9-10 rectangular regions spanning the diagonal to a two dimensional Lorentzian function, giving a precise measurement of the center of the spine. This was done for two separate two dimensional spectra; the errors appear to be random, with no evidence of systematic deviation. To confirm this, the center spine was fit to a straight line $v_{1}=m v_{2}+c$. The slope is statistically identical to 2.0 and the intercept to $0 \mathrm{kHz}$. A weighted combination of the two sets of measurements, 

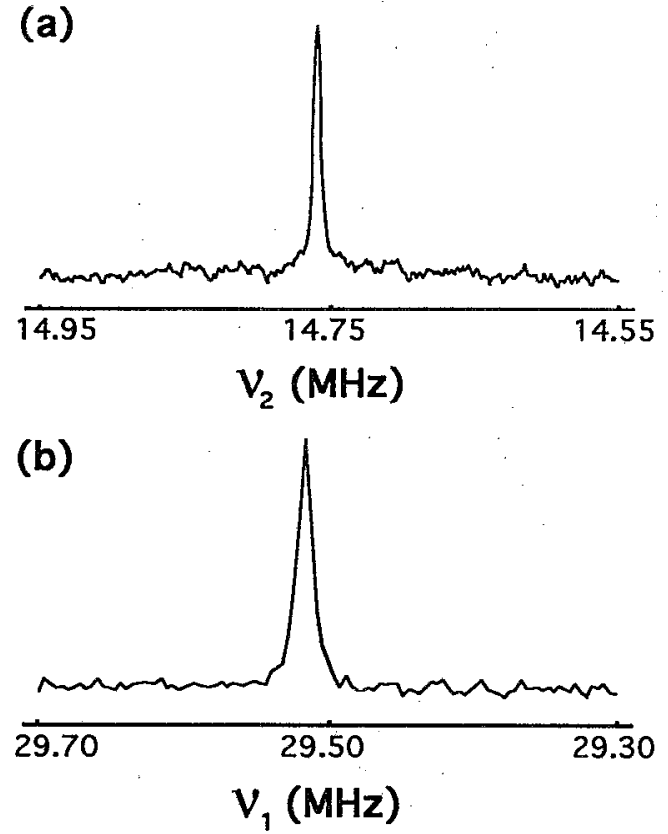

FIG. 4. Slices through the two dimensional zero-field NMR spectrum of ${ }^{127}$ I cadmium iodide. (a) The $(3 / 2-1 / 2)$ transition. (b) The $(5 / 2-3 / 2)$ transition.

using $t$ distribution of $95 \%$ confidence, gives a confidence interval value of $\left(v_{1}-2 v_{2}\right)=0.047 \pm 0.113 \mathrm{kHz}$; insertion in Eq. (5) finally gives a hexadecapole coupling constant of $0.216 \pm 0.514 \mathrm{kHz}$ which is indistinguishable from zero.

\section{LATTICE CALCULATIONS ON $\mathrm{CdI}_{2}$ AND KI}

The electric field gradient (EFG) and its second derivative were calculated for the cadmium and both iodide sites in $\mathrm{CdI}_{2}$, initially using the coordinates from the x-ray structure of Mitchell for the $4 \mathrm{H}$ polymorph. ${ }^{9}$ The computations were done in three stages; in the first stage, the electric field arising from each point charge in the lattice was calculated, and summed over a spherical region bounded on the outside by a hexagonal prism encompassing 136 unit cells in the $c$ direction and 250 unit cells in the $a$ direction. Use of a spherical region allowed us to circumvent the use of Ewald sums; nonetheless convergence was fairly slow. Since the crystals were leaflets, the Lorentz field contribution was expected to be negligible; calculations indeed showed that inclusion of a Lorentz field contribution had no significant effect on the computed field gradients.

Using this monopole field, the induced dipoles were calculated. The isotropic polarizability of $\mathrm{Cd}^{2+}$ was assumed to be $0.790 \times 10^{-30} \mathrm{~m}^{3} ;{ }^{10}$ the refractive indices $n_{\infty}$ have been measured as 2.08 (extraordinary) and 2.26 (ordinary). ${ }^{11}$ Using these values, together with the ClausiusMossotti relation, we calculated the principal values of the electric dipolar polarizability for $\mathrm{I}^{-}$in $\mathrm{CdI}_{2}$ to be $\alpha_{\|}$ $=6.288 \times 10^{-30} \mathrm{~m}^{3}, \alpha_{1}=6.951 \times 10^{-30} \mathrm{~m}^{3}$; since the crystal symmetry dictates that the electric field is directed purely along the $z$ direction, only $\alpha_{\|}$has any bearing on
TABLE I. Calculated electric field gradients at the cadmium and iodide sites in cadmium iodide.

\begin{tabular}{lllc}
\hline \hline & \multicolumn{3}{c}{ Calculated $\nabla E\left(\times 10^{19} \mathrm{~V} \mathrm{~m}^{-2}\right)$} \\
\cline { 2 - 4 } Site & Cadmium & Iodide (I) & Iodide (II) \\
\hline 2H & -5.6055 & -0.0830 & 1.4485 \\
$4 \mathrm{H}$ & -5.7221 & -1.0774 & 2.2125 \\
Expt. & $\pm 1.7196^{\mathrm{a}}$ & $\pm 3.5553^{\mathrm{b}}$ & $\pm 3.6079^{\mathrm{b}}$ \\
\hline \hline
\end{tabular}

Reference 14.

${ }^{b}$ Present work.

the present work. Several cycles of iteration generally sufficed to make the dipole field converge, whereupon the quadrupolar polarizabilities were introduced: These were taken as $0.788 \times 10^{-50} \mathrm{~m}^{5}$ for $\mathrm{Cd}^{2+}$ ion and $15.54 \times 10^{-50}$ $\mathrm{m}^{5}$ for $\mathrm{I}^{-}$ion. These values were assumed to be isotropic, and are taken from theoretical estimates in the literature. ${ }^{12}$ They are likely to be highly inaccurate; but the ultimate effect on the $\mathbf{\nabla E}$ was small and on $\nabla^{3} \mathbf{E}$ negligible. The $\mathbf{E}$ and $\nabla E$ at each lattice site were adjusted iteratively until they converged. At this point final values for $\nabla \mathbf{E}$ and $\nabla^{3} \mathbf{E}$ at the three distinct sites were computed. The computed EFG values for the ${ }^{111} \mathrm{Cd}$ and the two ${ }^{127} \mathrm{I}$ sites in cadmium iodide for the major $4 \mathrm{H}$ modification and $2 \mathrm{H}$ modification are shown in Table I. Since the quadrupole coupling constant can be obtained from the frequencies observed using Eq. (5), with a Sternheimer factor of -162.42 and a quadrupole moment of $-0.69 \times 10^{-28} \mathrm{~m}^{2}$ for the iodide ion, the electric field gradient of the iodide ion can be calculated using Eq. (7). ${ }^{13}$ Similarly, a Sternheimer factor of -29.3 and a quadrupole moment of $0.77 \times 10^{-28} \mathrm{~m}^{2}$ were used for the $\mathrm{Cd}^{2+}$ ion. ${ }^{14}$ The measured EFG values, from previous ${ }^{127}$ I zero-field NMR and perturbed angular correlation results for the spin $5 / 2$ excited nuclear state of ${ }^{111} \mathrm{Cd}$, are also given in Table $\mathrm{I}$.

Calculated $\nabla^{3} \mathbf{E}$ values for $\mathrm{KI}$ required inclusion of only the monopole field, since by symmetry the dipole and quadrupole parts of the electric potential at both the $\mathrm{K}$ and I sites are identically zero. The computed second derivative of the EFG values for $\mathrm{CdI}_{2}$ and $\mathrm{KI}$ are given in Table II. It can be seen that the hexadecapole interaction of iodine in $\mathrm{CdI}_{2}$ should be about $50 \%$ greater than that in KI. Since hexadecapole coupling constants can be calculated from the two dimensional ${ }^{127}$ I zero-field NMR correlation spectrum, the product $\left(1-\eta_{\infty}\right) e H$ of Sternheimer-amplified nuclear hexadecapole moment of iodide ion also can be obtained using Eq. (8).

The comparison in Table I between the measured zerofield NMR frequencies for $\mathrm{CdI}_{2}$ and those calculated by us at first glance appears to show very poor agreement. In

TABLE II. The second derivative of calculated electric field gradient of iodide site in cadmium iodide and potassium iodide.

\begin{tabular}{lcc}
\hline \hline & Calculated $\nabla^{3} \mathrm{E}\left(10^{40} \mathrm{~V} \mathrm{~m}^{-4}\right)$ \\
\hline Iodide in KI & Iodide (I) in $\mathrm{CdI}_{2}$ & Iodide (II) in $\mathrm{CdI}_{2}$ \\
-2.24042 & 3.37037 & 3.54286 \\
\hline \hline
\end{tabular}


fact, the agreement is much better than it appears. Because the $\mathrm{CdI}_{2}$ structure approaches hexagonal close packing of iodides, for which the EFG tensors would be identically zero for all sites, the nuclear quadrupole couplings are unusually small, and small changes in lattice dimensions cause proportionately enormous changes in the calculated zero-field NMR frequencies. At this point one must question how accurate the x-ray structure of $\mathrm{CdI}_{2}$ actually are. The earliest structure, of the comparatively simple $2 \mathrm{H}$ modification, gave a value of the lattice parameter $u$ which defines the $z$ axis position of the iodines within the unit cell as $0.25 .^{15}$ This value was based solely on the inability of the investigator to detect differences between pairs of reflections which would be identical at $u=0.25$ and different otherwise. However, given the earliness of the study and the fact that visual estimation of film data was being used, it is difficult to gauge the precision with which the parameter was determined. Unfortunately, in the standard compilation of crystal structures by Wyckoff, ${ }^{16} 0.25$ was erroneously transcribed as $1 / 4$, which carries the implication that the value of $u$ is constrained by the symmetry of a special position, which is indubitably not the case; for example, the isomorphous structures of $\mathrm{PbI}_{2}, \mathrm{MnI}_{2}$, and $\mathrm{NiI}_{2}$, show $u$ values of $0.245,0.265$, and 0.220 , respectively. ${ }^{16-18}$ However, in all of the later literature, this fractional atomic position has been carried over as a fixed parameter, and so it is unclear by how much $u$ actually might vary from 0.250 .

We have therefore examined the dependence of the computed EFG tensor on the lattice parameters $u$ and $v$ of the major $4 \mathrm{H}$ modification in cadmium iodide; these values are plotted in Fig. 5. The EFG value for each site depends on both $u$ and $v$; however, the EFG value of the iodide (I) site is relatively insensitive to $v$, and that of iodide (II) site to $u$. From Fig. 5, it can be seen that perfect agreement between calculation and experiment can be obtained for the iodide (I) site with a deviation of as little as 0.007 of a unit cell (or $0.10 \AA$ ) in the iodine positions; and for the iodide (II) site with a deviation of as little as 0.003 of a unit cell (or $0.04 \AA$ ) in the iodine positions.

In the region near the reported values of the lattice parameters $u$ and $v$, the calculated EFG forms a nearly planar surface. We therefore fit the computed EFG values of each site to a plane using linear least squares methods. These planes are shown in Fig. 6. They intersect the experimental EFG values for the three atomic sites along three lines, as shown in Fig. 7. These lines define the region which contains the most likely values of the lattice parameters $u$ and $v$, bringing the computed nuclear quadrupole coupling constant into good agreement with experiment. The small change in lattice parameters is probably within the margin of error of crystallographic studies.

\section{DISCUSSION}

\section{The reliability of lattice calculations}

The disagreement between zero-field NMR results and calculated field gradients has been rationalized in various ways in the past; however, since clearly a very minor (a)

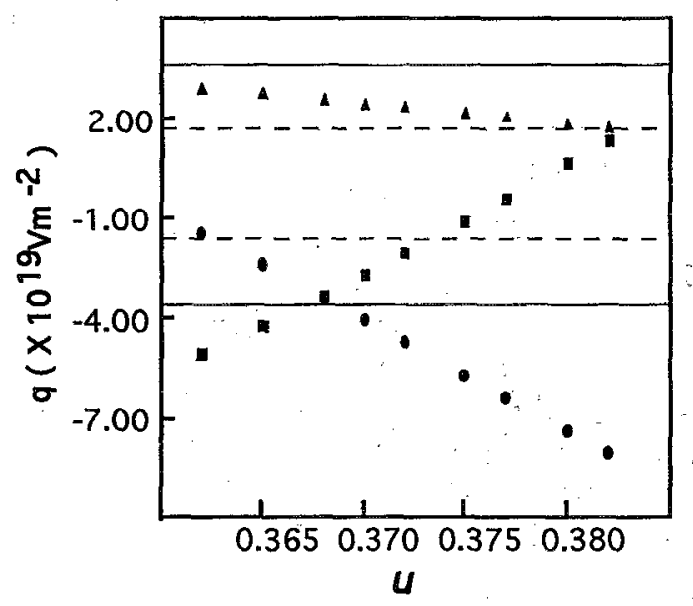

(b)

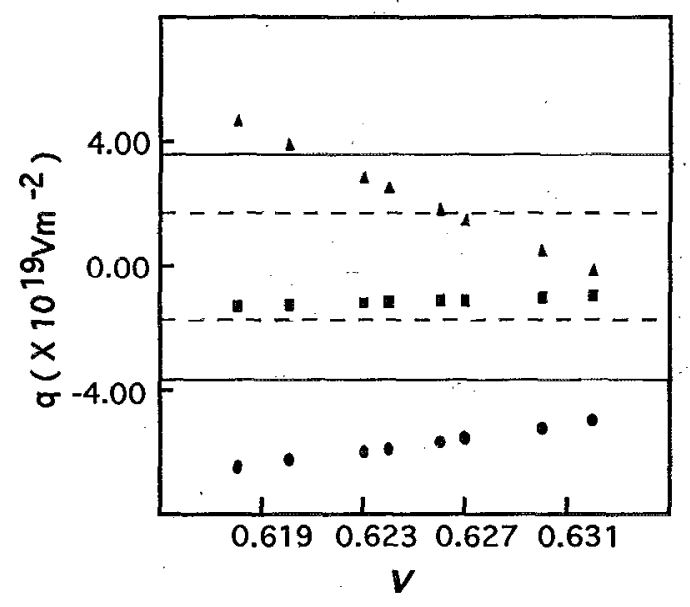

FIG. 5. The computed EFG values of both cadmium and iodide sites for the $4 \mathrm{H}$ modification of cadmium iodide, plotted against the values of the lattice parameters (a) $u$ and (b) $v$. The dashed and solid lines represent the experimental values of EFG tensor for cadmium and iodide sites. $\square$, $\Delta$, and - represent the computed EFG tensor for iodide (I) site, iodide (II) site and cadmium site respectively. Details are described in the text.

change in the atomic positions is needed to bring about good agreement with the zero-field NMR data, and since the accuracy of the $x$-ray determinations until now is questionable, we believe that a redetermination of the $\mathrm{CdI}_{2}$ structure is called for. Other sources of error in the calculated results may lie in inaccuracies in the electronic shielding and antishielding terms. While the dipolar polarizabilities are probably fairly reliable-that of $\mathrm{Cd}^{2+}$ is small, and that of $\mathrm{I}^{-}$may be estimated with reasonable confidence from the Clausius-Mossotti relation, and the Sternheimer factors can be assumed to be accurate to about $10 \%-20 \%$, the quadrupolar polarizabilities have no experimental basis, and those of iodide are probably strongly dependent on lattice environment. Fortunately, the contribution of the quadrupolar polarizability to the overall EFG tensor is 


\section{(a)}

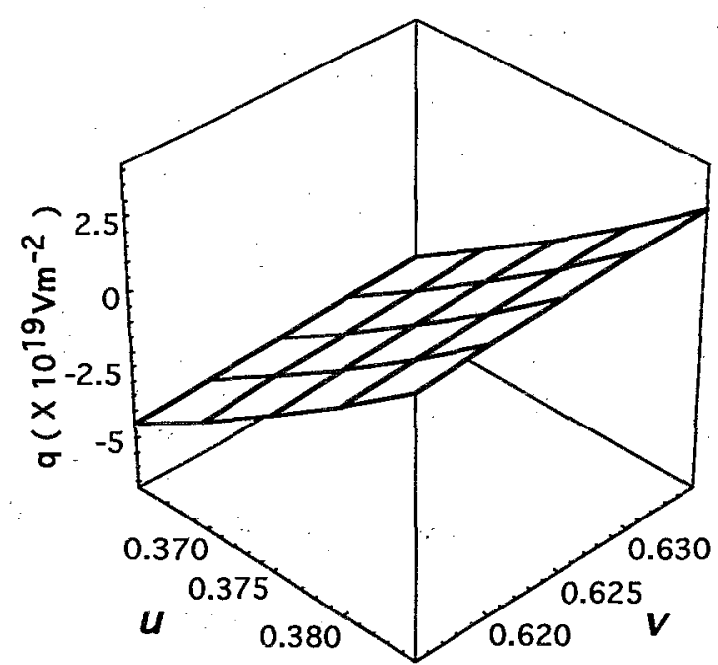

(b)

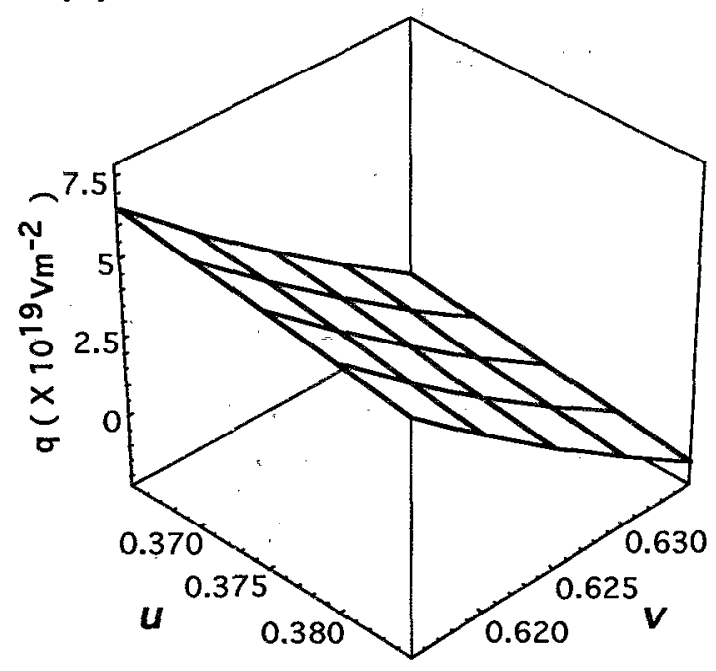

(c)

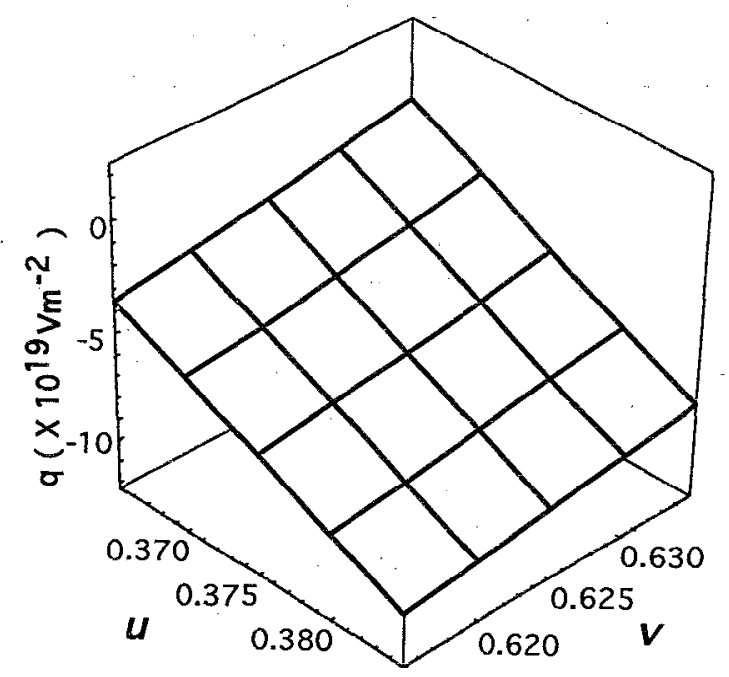

FIG. 6. Surface plot of the computed EFG of each ion site against both $u$ and $v$ for the $4 \mathrm{H}$ modifications of cadmium iodode: (a) Iodide (I) site. (b) Iodide (II) site. (c) Cadmium site.

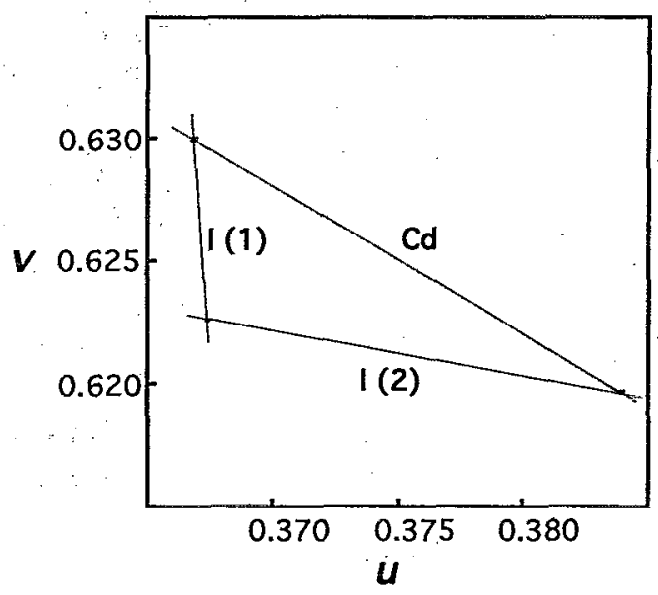

FIG. 7. Trajectories describing the best fit of calculated and experimental values of the EFG tensor as a function of the lattice parameters $u$ and $v$.

rather small; changing the quadrupolar polarizability of iodide or cadmium by a factor of 2 causes no more than $5 \%$ change in the EFG tensors.

\section{Upper limit on hexadecapole moment of ${ }^{127}$ I}

Most of these considerations are in any case moot from the standpoint of the hexadecapolar coupling. That coupling is nonzero for a hcp lattice, and so the tensors do not lie near a null; furthermore, the interaction is much more short range than the quadrupolar interaction, and so the effects of inaccuracies in the assumed polarizabilities are negligible. Therefore, the computed values for $\nabla^{3} \mathbb{E}$ are likely to be much more reliable than those for $\nabla \mathbf{E}$; particularly in the case of $\mathrm{KI}$, where calculated values do not depend at all on shielding effects of tensor rank lower than four.

We can calculate with reasonable accuracy the electric field gradient tensors of $\mathrm{CdI}_{2}$ at all three atomic positions despite the fact that these tensors are quite sensitive to the values of several uncertain parameters. $\nabla^{3} \mathrm{E}$ is expected to be much less sensitive to inaccurate assumptions about polarizabilities or lattice dimensions. Therefore, the major part of the uncertainty in the measurement of the nuclear hexadecapole moment of ${ }^{127} \mathrm{I}$ is experimental. Based on a measured value of $0.216 \pm 0.514 \mathrm{kHz}$ for iodine in $\mathrm{CdI}_{2}$, from two dimensional ${ }^{127}$ I zero-field NMR, we can set an upper limit of $0.730 \mathrm{kHz}$ on the nuclear hexadecapole coupling constant. In combination with computed values of the third derivative of the electric field at the iodide site, the calculated Sternheimer-amplified nuclear hexadecapole moment of ${ }^{127} \mathrm{I}$ is no larger than $8.96 \times 10^{-53} \mathrm{~m}^{4}$. This measurement disagrees markedly with the results of $\mathrm{Ni}$ and Sears ${ }^{6}$ who measured a nonzero hexadecapole coupling constant several times larger than our uncertainty.

Since the $\nabla^{3} \mathbf{E}$ in Eq. (12) is a rigorously calculated value, it is likely that the discrepancy lies in the measurement techniques. In this regard, we would like to emphasize the simplicity and directness of the two dimensional zero-field method, which measures the correlated free pre- 
cession frequencies of pairs of connected transitions, in contrast with the high-field NMR method, which relies on measurement of echo delays, which can be produced by a host of experimental factors besides hexadecapole moments. In particular, we note that the second order term in the electric quadrupolar Hamiltonian in the presence of a strong Zeeman field has a fourth rank part which has similar symmetry properties to the clectric hexadecapole coupling and is difficult to separate from it. Since the linewidths quoted by $\mathrm{Ni}$ and Sears $^{6}$ are those which would be expected from the excitation bandwidth of the spectrometer, it is difficult to gauge the true range of the quadrupole coupling constants in their crystals; however, even with the bandwidths they quote, fourth rank components of the order as their measured hexadecapolar frequencies can be expected. The authors discount these effects without elaboration.

\section{ACKNOWLEDGMENTS}

We thank Dr. Adilgiry Kusov and Raju Subramanian for informative discussions and suggestions. This research was supported by grants from the Procter and Gamble Company in conjunction with an NSF Presidential Young
Investigator Award to G.S.H. (DOB 9057765), by a Dreyfus Foundation Teacher Scholar Award (also To G.S.H.), and by the National Institutes of Health (GM-39071).

${ }^{1}$ T. C. Wang, Phys. Rev. 99, 566 (1955).

${ }^{2}$ R. R. Hewitt and B. F. Williams, Phys. Rev. 129, 1188 (1963).

${ }^{3}$ S. L. Segel, J. Chem. Phys. 69, 2434 (1978).

${ }^{4}$ H. Gotou, J. Magn. Reson. 54, 36 (1983).

${ }^{5}$ E. B. Doering and J. S. Waugh, J. Chem. Phys. 85, 1753 (1986).

${ }^{6}$ Q. W. Ni and R. E. J. Sears, J. Chem. Phys. 95, 4796 (1991).

${ }^{7}$ M.-Y. Lian and G. S. Harbison, J. Magn. Reson. 99, 198 (1992).

${ }^{8}$ V. S. Grechishkin and E. M. Shishkin, Opt. Spectrosc. 33, 319 (1972).

${ }^{9}$ R. S. Mitchell, Z. Krist. 108, 296 (1956).

${ }^{10}$ G. D. Mahan, Phys. Rev. A 22, 1780 (1980):

${ }^{11}$ (a) M. R. Tubbs, J. Phys. Chem. Solids 27, 1667 (1966); (b) P. A. Lee, G. Said, R. Davis, and T. H. Lim ibid. 30, 2719 (1969).

${ }^{12}$ P. C. Schmidt, A. Weiss, and T. P. Das, Phys. Rev. B 19, 5525 (1979).

${ }^{13}$ (a) P. C. Schmidt, K. D. Sen, T. P. Das, and A. Weiss, Phys. Rev. B 22, 4167 (1980); (b) K. Murakawa, Phys. Rev. 100, 1369 (1955).

${ }^{14}$ (a) H. Hass and D. A. Shirley, J. Chem: Phys. 58, 3339 (1973); (b) R. S. Raghavan, P. Raghavan, and J. M. Friedt, Phys. Rev. Lett. 30, 10 (1973).

${ }^{\text {Is }}$ R. M. Bozorth, J. Am. Chem. Soc. 44, 2232 (1922).

${ }^{16}$ R. W. G. Wyckoff, Crystal Structures, 2nd ed. (Wiley, New York, 1963), Vol. 1 .

${ }^{17}$ J. W. Cable, M. K. Wilkinson, E. O. Wollan, and W. C. Koehler, Phys. Rev. 126, 1860 (1962).

${ }^{18}$ M. Gaudin and D. Weigel, C. R. Acad. Sci. Paris B 264, 895 (1967). 